\title{
Analytical model of the response of a superconducting film to line currents
}

\author{
Yasunori Mawatari \\ National Institute of Advanced Industrial Science and Technology (AIST), Tsukuba, Ibaraki 305-8568, Japan \\ John R. Clem \\ Ames Laboratory-DOE and Department of Physics and Astronomy, Iowa State University, Ames Iowa 50011, USA
}

(Received July 3, 2006; Revised September 15, 2006)

\begin{abstract}
We theoretically investigate the response of a superconducting film to line currents flowing in linear wires placed above the film, and we present analytic expressions for the magnetic-field and current distributions based on the critical state model. The behavior of the superconducting film is characterized by the sheet-current density $K_{z}$, whose magnitude cannot exceed the critical value $j_{c} d$, where $j_{c}$ is the critical current density and $d$ is the thickness of the film. When the transport current $I_{0}$ flowing in the wire is small enough, $\left|K_{z}\right|$ is smaller than $j_{c} d$ and the magnetic field is shielded below the film. When $I_{0}$ exceeds a threshold value $I_{c 0} \propto j_{c} d$, on the other hand, $\left|K_{z}\right|$ reaches $j_{c} d$ and the magnetic field penetrates below the film. We also calculate the ac response of the film when an ac transport current flows in the linear wires.

PACS numbers: 74.25.Sv, 74.25.Nf, 74.78.-w
\end{abstract}

\section{INTRODUCTION}

The response of superconducting films to homogeneous applied magnetic fields is well understood, and analytic expressions for the distributions of the magnetic field and current density have been derived ${ }^{1,2,3,4,5,6}$ based on Bean's critical state model $\underline{7}$ When small currentcarrying coils are placed near the surface to probe the local properties of superconducting films, the magnetic fields generated by the coils are inhomogeneous. Analytic expressions describing the response of superconducting films to small coils have been derived for the linear response regime, $, 8,9,10,11,12,13$ but in order to measure the local distribution of the critical current density $j_{c}$ in superconducting films, it is necessary to investigate the nonlinear response 14.15.16.17.18 Numerical computations of the nonlinear response of superconducting films to the inhomogeneous magnetic fields arising from small coils have been carried out in Refs. 11.192021.22, but here we present analytic results for the nonlinear response to line currents above superconducting films.

The procedure proposed by Claassen et al 15 for inductive measurements of the local $j_{c}$ distribution in films of thickness much greater than the London penetration depth $\lambda$ can be described briefly as follows. A small coil carrying a sinusoidal drive current $I_{0} \cos \omega t$ is placed just above a superconducting film, and the induced voltage $V(t)=\sum_{n} V_{n} \cos \left(n \omega t+\vartheta_{n}\right)$ in the coil is detected. The amplitude of the third-harmonic voltage $V_{3}$ is measured as a function of the drive current amplitude $I_{0}$, and the threshold current $I_{c 0}$ is defined such that $V_{3}=0$ for $0<I_{0}<I_{c 0}$ and $V_{3}>0$ for $I_{0}>I_{c 0}$. Because $I_{c 0} \propto j_{c} d$, where $d$ the film thickness, $j_{c}$ can be evaluated from $I_{c 0}, 15,16,18$ Since an electric-field criterion must be applied for a precise determination of $j_{c}$, it is important to evaluate the electric field $E_{f}$ generated in the superconducting film. 23

In the present paper we consider linear wires as simple models of coil wires, and we analytically investigate the response of a superconducting film to linear wires carrying transport currents. In Sec. II we investigate the dc (ac) response of a superconducting film to a linear wire carrying a dc (ac) transport current: we determine the threshold current $I_{c 0}$, and we present the voltage $V(t)$ and the harmonic voltages induced in the linear wire, as well as the electric field $E_{f}$ induced in the superconducting film. In Sec. III we consider a superconducting film and two linear wires carrying transport currents of opposite directions. We briefly summarize our results in Sec. V

\section{A SINGLE LINEAR WIRE AND A SUPERCONDUCTING FILM}

In this section we consider a superconducting film and a linear wire carrying a transport current, as shown in Fig. 11 An infinitely long wire, parallel to the $z$ axis, is situated at $(x, y)=\left(0, y_{0}\right)$ where $y_{0}>0$. The radius of the wire $r_{w}$ is assumed to be much smaller than $y_{0}$. A superconducting film, infinitely extended in the $x z$ plane, is situated at $-d / 2<y<+d / 2$, where the film thickness $d$ is much smaller than $y_{0}$ but is larger than the

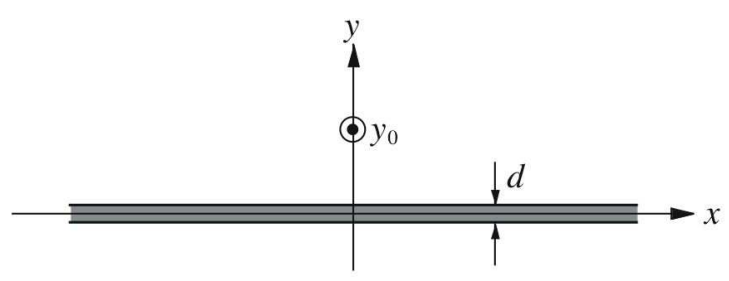

FIG. 1: Configuration of a superconducting film at $y=0$ and a linear wire at $(x, y)=\left(0, y_{0}\right)$. The film is infinitely extended in the $x z$ plane, and the infinite wire is parallel to the $z$ axis. 
London penetration depth $\lambda$. Flux pinning in the film is characterized by the critical current density $j_{c}$, which is assumed to be constant (independent of magnetic field) as in Bean's critical state model ${ }^{7}$ and to be spatially homogeneous in the film. We consider the limit $d \rightarrow 0$, as this simplification allows us to obtain simple analytic expressions for the magnetic-field and current distributions. In the thin-film limit of $d \rightarrow 0$, the sheet current $K_{z}(x)=\int_{-d / 2}^{+d / 2} j_{z}(x, y) d y$ plays crucial roles, and the upper limit of $\left|K_{z}\right|$ is the critical sheet-current density $j_{c} d$. The lower critical field $H_{c 1}$ is assumed to be much smaller than $j_{c} d$ (i.e., $H_{c 1} / j_{c} d \rightarrow 0$ ), such that the flux penetration into superconducting films is not affected by $H_{c 1}$, but is determined by $j_{c} d{ }^{24}$

We introduce the complex field $\mathcal{H}(\zeta)=H_{y}(x, y)+$ $i H_{x}(x, y), \stackrel{25.26 .27}{w}$ which is an analytic function of $\zeta=$ $x+i y$ for $y \neq 0$ and $(x, y) \neq\left(0, y_{0}\right)$. The Biot-Savart law for the complex field is given by

$$
\mathcal{H}(\zeta)=\mathcal{H}_{0}(\zeta)+\frac{1}{2 \pi} \int_{-\infty}^{+\infty} d u \frac{K_{z}(u)}{\zeta-u}
$$

where $\mathcal{H}_{0}(\zeta)$ is the complex field arising from the line current alone. The $\mathcal{H}_{0}(\zeta)$ is given by

$$
\mathcal{H}_{0}(\zeta)=\frac{I_{z}}{2 \pi} \frac{1}{\zeta-i y_{0}},
$$

where $I_{z}$ is the transport current flowing in the linear wire. At the upper $(\zeta=x+i \epsilon)$ and lower $(\zeta=x-i \epsilon)$ surfaces of the superconducting film, where $\epsilon=d / 2$ is a positive infinitesimal, the perpendicular and parallel magnetic fields $H_{y}(x, 0)=\operatorname{Re} \mathcal{H}(x \pm i \epsilon)$ and $H_{x}(x, \pm \epsilon)=$ $\operatorname{Im} \mathcal{H}(x \pm i \epsilon)$ are obtained from Eq. (11) as

$$
\begin{aligned}
H_{y}(x, 0) & =\operatorname{Re}_{0}(x)+\frac{1}{2 \pi} \mathrm{P} \int_{-\infty}^{+\infty} d u \frac{K_{z}(u)}{x-u}, \\
H_{x}(x, \pm \epsilon) & =\operatorname{Im} \mathcal{H}_{0}(x) \mp K_{z}(x) / 2
\end{aligned}
$$

where $\mathrm{P}$ denotes the Cauchy principal value integral. The complex potential is defined by $\mathcal{G}(\zeta)=\int \mathcal{H}(\zeta) d \zeta$, and the contour lines of the real part of $\mathcal{G}(\zeta)$ correspond to magnetic-field lines.

The magnetic flux per unit length $\Phi_{w}$ around the linear wire is

$$
\begin{aligned}
\Phi_{w} & =-\mu_{0} \int_{y_{0}+r_{0}}^{\infty} d y H_{x}(0, y) \\
& =\mu_{0} \operatorname{Re}\left[-\mathcal{G}\left(i\left(y_{0}+r_{0}\right)\right)+\lim _{v \rightarrow \infty} \mathcal{G}(i v)\right] .
\end{aligned}
$$

We have introduced a cutoff length $r_{0} \ll y_{0}$, where $r_{0}$ is of the order of the radius of the wire, to remove the logarithmic divergence as $\zeta \rightarrow i y_{0}$. The magnetic flux per unit length $\Phi_{f}\left(x^{\prime}\right)$ up through the film $(y=0)$ in the region $x^{\prime}<x<+\infty$ is

$$
\begin{aligned}
\Phi_{f}\left(x^{\prime}\right) & =\mu_{0} \int_{x^{\prime}}^{\infty} d u H_{y}(u, 0) \\
& =\mu_{0} \operatorname{Re}\left[-\mathcal{G}\left(x^{\prime}\right)+\lim _{u \rightarrow \infty} \mathcal{G}(u)\right] .
\end{aligned}
$$

\section{A. DC response}

In this subsection we consider the magnetic-field distribution when the linear wire carries a dc current $I_{z}=$ $I_{0}>0$ that is held constant after monotonically increasing from $I_{z}=0$.

\section{Linear response for $0<I_{0} \leq I_{c 0}$}

For $0<I_{0} \leq I_{c 0}$, the magnetic field is completely shielded below the film, $y=\operatorname{Im} \zeta<0$. The field distribution can be obtained by the mirror-image technique, and the resulting complex field is

$$
\mathcal{H}(\zeta)= \begin{cases}\frac{I_{0}}{\pi} \frac{i y_{0}}{\zeta^{2}+y_{0}^{2}} & \text { for } \operatorname{Im} \zeta>0, \\ 0 & \text { for } \operatorname{Im} \zeta<0\end{cases}
$$

The complex potential $\mathcal{G}(\zeta)=\int \mathcal{H}(\zeta) d \zeta$ for $\operatorname{Im} \zeta>0$ is given by

$$
\mathcal{G}(\zeta)=\frac{I_{0}}{\pi} i \arctan \left(\frac{\zeta}{y_{0}}\right) .
$$

The perpendicular magnetic field and sheet-current density are thus given by $H_{y}(x, 0)=0$ and

$$
K_{z}(x)=-\frac{I_{0}}{\pi} \frac{y_{0}}{x^{2}+y_{0}^{2}},
$$

respectively. The net current induced in the superconducting film is $\int_{-\infty}^{+\infty} K_{z}(x) d x=-I_{0}$, as expected. Note that the sheet-current density cannot exceed $j_{c} d$; that is, $\left|K_{z}(x)\right| \leq j_{c} d$. Because the maximum of $\left|K_{z}\right|$ given by Eq. (9) is $I_{0} / \pi y_{0}$, Eq. (9) is valid for $I_{0} \leq I_{c 0}$, where the threshold current is given by

$$
I_{c 0}=\pi j_{c} d y_{0} .
$$

Figure 2(a) shows the magnetic-field lines [i.e., the contour lines of $\operatorname{Re} \mathcal{G}(x+i y)$ ] calculated from Eq. [8], and the dashed line in Fig. 2(d) shows $K_{z}(x)$ given by Eq.(9).

The magnetic flux per unit length around the linear wire, calculated by substituting Eq. (7) into Eq. (5), is

$$
\Phi_{w}=\frac{\mu_{0} I_{0}}{\pi} \int_{y_{0}+r_{0}}^{\infty} d y \frac{y_{0}}{y^{2}-y_{0}^{2}}=L_{0} I_{0}
$$

where the inductance per unit length $L_{0}=$ $\left(\mu_{0} / 2 \pi\right) \ln \left(2 y_{0} / r_{0}\right)$ corresponds to the difference between the self inductance of the linear wire and the mutual inductance of the wire and its image. Because the perpendicular magnetic field in the film is zero, the magnetic flux up through the film defined by Eq. (6) is also zero: ${ }^{28}$

$$
\Phi_{f}(x)=0 .
$$

Equations (7), (8), (9), (11), and (12) are valid for $0<I_{0} \leq I_{c 0}$. 


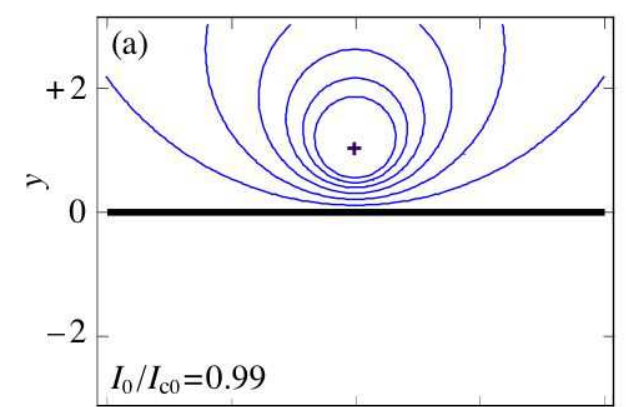

(d)

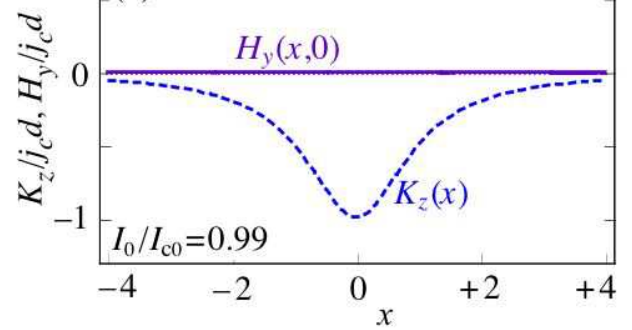

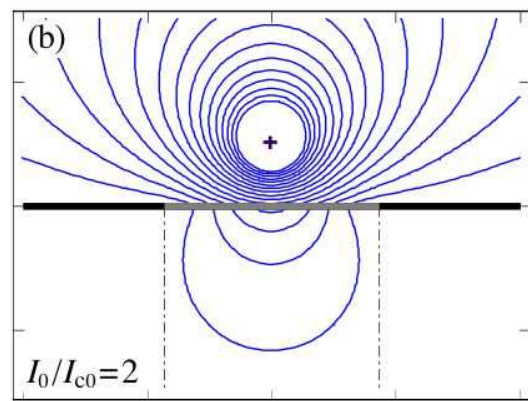

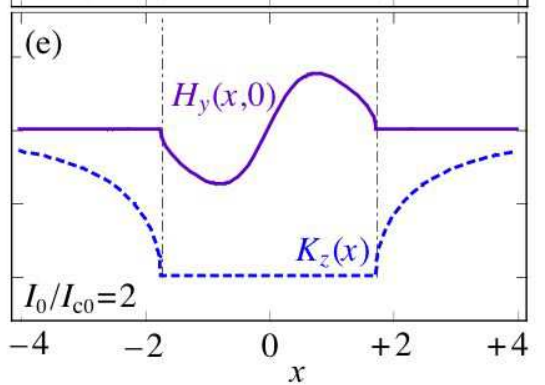

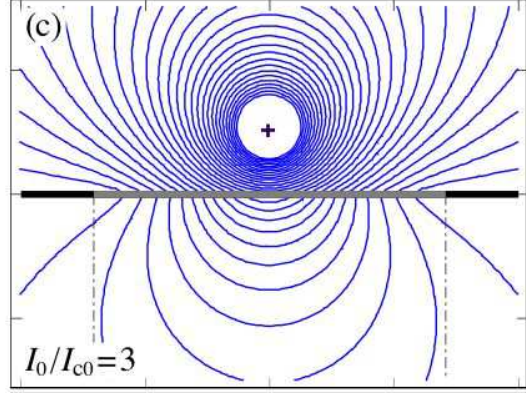

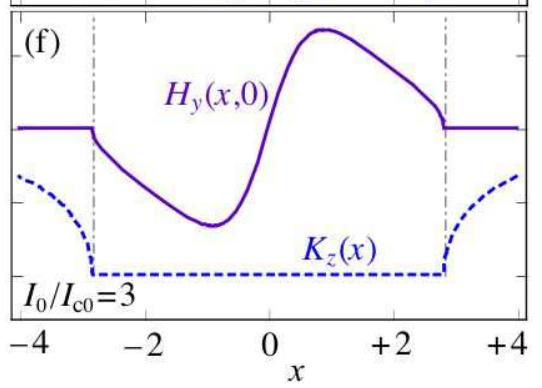

FIG. 2: (color online) Magnetic-field and sheet-current distributions (a,d) for $I_{0} / I_{c 0}=0.99$, (b,e) for $I_{0} / I_{c 0}=2(a=1.73)$, and $(\mathrm{c}, \mathrm{f})$ for $I_{0} / I_{c 0}=3(a=2.83)$. Top figures $(\mathrm{a}, \mathrm{b}, \mathrm{c})$ show the magnetic-field lines around a linear wire and a superconducting film. The cross symbols at $(x, y)=\left(0, y_{0}\right)=(0,1)$ denote the position of the linear wire, the black horizontal line the shielded region $|x|>a$ of the superconducting film, and the gray horizontal lines the penetrated region $|x|<a$ of the film. The vertical dot-dashed lines indicate the positions of the flux front at $x= \pm a$. Bottom figures (d,e,f) show the distributions of the perpendicular magnetic field $H_{y}(x, 0)$ divided by $j_{c} d$ (solid lines) and the sheet-current density $K_{z}(x)$ divided by $j_{c} d$ (dashed lines) in the superconducting film.

\section{Nonlinear response for $I_{0}>I_{c 0}$}

For $I_{0}>I_{c 0}$, on the other hand, the maximum $\left|K_{z}\right|$ reaches $j_{c} d$ and the magnetic field penetrates below the superconducting film. The field distributions for $I_{0}>$ $I_{c 0}$, therefore, must satisfy

$$
\begin{aligned}
& K_{z}(x)=-j_{c} d \text { and } H_{y}(x, 0) \neq 0 \text { for }|x|<a \text {, } \\
& \left|K_{z}(x)\right|<j_{c} d \text { and } H_{y}(x, 0)=0 \text { for }|x|>a \text {, }
\end{aligned}
$$

where the flux fronts [i.e., the boundaries between the region of $H_{y}(x, 0) \neq 0$ and that of $H_{y}(x, 0)=0$ ] are at $x= \pm a$. The complex field and the parameter $a$ are determined such that they are consistent with Eqs. (13) and (14), as derived in Appendix $\mathrm{A}$ The parameter $a$ is a function of $I_{0}$,

$$
a=y_{0} \sqrt{\left(I_{0} / I_{c 0}\right)^{2}-1},
$$

and the complex field is

$$
\mathcal{H}(\zeta)=\frac{j_{c} d}{2}\left( \pm i+\frac{i y_{0} \sqrt{a^{2}+y_{0}^{2}}+\zeta \sqrt{a^{2}-\zeta^{2}}}{\zeta^{2}+y_{0}^{2}}\right)
$$

where the upper sign holds for $\operatorname{Im} \zeta>0$ and the lower sign for $\operatorname{Im} \zeta<0$. The corresponding complex potential is

$$
\begin{aligned}
\mathcal{G}(\zeta)= & \frac{j_{c} d}{2}\left\{ \pm i \zeta+\sqrt{a^{2}-\zeta^{2}}+\sqrt{a^{2}+y_{0}^{2}}\right. \\
& \left.\times\left[i \arctan \left(\frac{\zeta}{y_{0}}\right)-\operatorname{arctanh}\left(\sqrt{\frac{a^{2}-\zeta^{2}}{a^{2}+y_{0}^{2}}}\right)\right]\right\} .
\end{aligned}
$$

Figures 2(b) and (c) show the magnetic-field lines, i.e., the contour lines of $\operatorname{Re} \mathcal{G}(x+i y)$ calculated from Eq. (17).

The perpendicular magnetic field $H_{y}(x, 0)=\operatorname{Re} \mathcal{H}(x \pm$ $i \epsilon)$ in the superconducting film, obtained from Eq. (16), is

$$
H_{y}(x, 0)= \begin{cases}\frac{j_{c} d}{2} \frac{x \sqrt{a^{2}-x^{2}}}{x^{2}+y_{0}^{2}} & \text { for }|x|<a, \\ 0 & \text { for }|x|>a,\end{cases}
$$

and the sheet-current density $K_{z}(x)=\operatorname{Im}[\mathcal{H}(x-i \epsilon)-$ $\mathcal{H}(x+i \epsilon)]$ is

$$
K_{z}(x)= \begin{cases}-j_{c} d & \text { for }|x|<a, \\ -j_{c} d\left(1-\frac{|x| \sqrt{x^{2}-a^{2}}}{x^{2}+y_{0}^{2}}\right) & \text { for }|x|>a .\end{cases}
$$

The net current induced in the superconducting film is again $\int_{-\infty}^{+\infty} K_{z}(x) d x=-I_{0}$, as expected. Figures $2(\mathrm{e})$ 
and (f) show $H_{y}(x, 0)$ and $K_{z}(x)$ given by Eqs. (18) and (19), respectively.

The magnetic flux per unit length around the linear wire, calculated by substituting Eq. (17) into Eq. (5), is

$$
\Phi_{w} \simeq-\frac{\mu_{0} j_{c} d}{2}\left[y_{0}-\sqrt{a^{2}+y_{0}^{2}}+\sqrt{a^{2}+y_{0}^{2}} \ln \left(\frac{2 \sqrt{a^{2}+y_{0}^{2}}}{r_{0}}\right)\right]
$$

Combining Eqs. (11), (15), and (20) yields $\Phi_{w}=\widetilde{\Phi}_{w}\left(I_{0}\right)$, where

$$
\widetilde{\Phi}_{w}\left(I_{0}\right)= \begin{cases}L_{0} I_{0} & \text { for } 0<I_{0} \leq I_{c 0}, \\ L_{0} I_{0}+\frac{\mu_{0}}{2 \pi}\left[I_{c 0}-I_{0}+I_{0} \ln \left(\frac{I_{0}}{I_{c 0}}\right)\right] & \text { for } I_{0}>I_{c 0} .\end{cases}
$$

The magnetic flux up through the film, calculated by substituting Eq. (17) into Eq. (6), is

$$
\Phi_{f}(x)=\frac{\mu_{0} j_{c} d}{2}\left[-\sqrt{a^{2}-x^{2}}+\sqrt{a^{2}+y_{0}^{2}} \operatorname{arctanh}\left(\sqrt{\frac{a^{2}-x^{2}}{a^{2}+y_{0}^{2}}}\right)\right]
$$

for $|x|<a$, and $\Phi_{f}(x)=0$ for $|x| \geq a$. Combining Eqs. (12), (15), and (22) yields $\Phi_{f}(x)=\widetilde{\Phi}_{f}\left(x, I_{0}\right)$, where

$$
\widetilde{\Phi}_{f}\left(x, I_{0}\right)= \begin{cases}0 & \text { for } 0<I_{0} \leq I_{c 0} \text { or }|x| \geq a, \\ \frac{\mu_{0}}{2 \pi}\left[\sqrt{I_{0}^{2}-I_{c 0}^{2}\left(1+\frac{x^{2}}{y_{0}^{2}}\right)}+I_{0} \operatorname{arccosh}\left(\frac{I_{0}}{I_{c 0}} \frac{y_{0}}{\sqrt{x^{2}+y_{0}^{2}}}\right)\right] & \text { for } I_{0}>I_{c 0} \text { and }|x|<a .\end{cases}
$$

\section{B. AC response}

In this subsection we consider the time-dependent field distributions when the linear wire carries a sinusoidal ac drive current $I_{z}(t)=I_{0} \cos \omega t$. In inductive measurements of the local $j_{c}$ in superconducting films, harmonic voltages induced in coils are detected. ${ }^{15,16,18}$ For precise $j_{c}$ measurements, it also is important to determine the electric field induced in the film. ${ }^{23}$

We wish to calculate the magnetic field around the linear wire $\Phi_{w}(t)$, defined by Eq. (5), and the voltage per unit length induced in the wire,

$$
V(t)=R_{w} I_{0} \cos \omega t-d \Phi_{w}(t) / d t
$$

where $R_{w}$ is the resistance per unit length of the wire. We also wish to determine the magnetic flux per unit length up through the film $\Phi_{f}(x, t)$, defined by Eq. (6), and the electric field in the film, ${ }^{29}$

$$
E_{f}(x, t)=-\partial \Phi_{f}(x, t) / \partial t
$$

$$
\text { 1. Linear response for } 0<I_{0} \leq I_{c 0}
$$

For $0<I_{0} \leq I_{c 0}$ the magnetic field is completely shielded below the superconducting film, $y=\operatorname{Im} \zeta<0$, as in Sec. IA 1 The complex field, the sheet-current density in the film, and the magnetic flux around the linear wire are given by Eqs. (7), (91), and (11), respectively, except that now $I_{0}$ in those equations is replaced by $I_{0} \cos \omega t$.

The magnetic flux per unit length around the linear wire is given by $\Phi_{w}(t)=L_{0} I_{0} \cos \omega t$. The voltage induced in the wire defined by Eq. (24) is thus given by $V(t)=R_{w} I_{0} \cos \omega t+\omega L_{0} I_{0} \sin \omega t$. For $0<I_{0} \leq I_{c 0}$, the harmonic voltages, the magnetic flux per unit length penetrating the film $\Phi_{f}$, and the electric field in the film $E_{f}$ are all zero. ${ }^{28}$

\section{Nonlinear response for $I_{0}>I_{c 0}$}

For $I_{0}>I_{c 0}$, on the other hand, the magnetic field penetrates through the superconducting film, as discussed in Sec. II A 2

For ac drive current $I_{0} \cos \omega t$, the magnetic flux per unit length around the linear wire $\Phi_{w}(t)$ is $\frac{4}{\underline{ }}$

$$
\Phi_{w}(t)=\widetilde{\Phi}_{w}\left(I_{0}\right)-2 \widetilde{\Phi}_{w}\left(I_{0}(1-\cos \omega t) / 2\right)
$$

for $0<\omega t<\pi$, and $\Phi_{w}(t)=-\Phi_{w}(t-\pi / \omega)$ for $\pi<\omega t<$ $2 \pi$, where $\widetilde{\Phi}_{w}\left(I_{0}\right)$ is defined by Eq. (21). The voltage per unit length of the wire, calculated from Eqs. (24) and 
(26), is

$$
\begin{aligned}
V(t)= & R_{w} I_{0} \cos \omega t \\
& +\omega I_{0} \sin \omega t \widetilde{L}_{w}\left(I_{0}(1-\cos \omega t) / 2\right),
\end{aligned}
$$

where $\widetilde{L}_{w}\left(I_{0}\right) \equiv d \widetilde{\Phi}_{w}\left(I_{0}\right) / d I_{0}$ is the differential inductance given by

$$
\widetilde{L}_{w}\left(I_{0}\right)= \begin{cases}L_{0} & \text { for } 0<I_{0}<I_{c 0}, \\ L_{0}+\frac{\mu_{0}}{2 \pi} \ln \left(\frac{I_{0}}{I_{c 0}}\right) & \text { for } I_{0}>I_{c 0} .\end{cases}
$$

In response to the ac drive current, the magnetic flux per unit length up through the film $\Phi_{f}(x, t)$ is

$$
\Phi_{f}(x, t)=\widetilde{\Phi}_{f}\left(x, I_{0}\right)-2 \widetilde{\Phi}_{f}\left(x, I_{0}(1-\cos \omega t) / 2\right)
$$

for $0<\omega t<\pi$, and $\Phi_{f}(x, t)=-\Phi_{f}(x, t-\pi / \omega)$ for $\pi<\omega t<2 \pi$, where $\widetilde{\Phi}_{f}\left(x, I_{0}\right)$ is defined by Eq. (23). The electric field induced in the film, calculated from Eqs. (25) and (29), is

$$
E_{f}(x, t)=\omega I_{0} \sin \omega t \widetilde{L}_{f}\left(x, I_{0}(1-\cos \omega t) / 2\right),
$$

where the function $\widetilde{L}_{f}\left(x, I_{0}\right) \equiv \partial \widetilde{\Phi}_{f}\left(x, I_{0}\right) / \partial I_{0}$ is given by

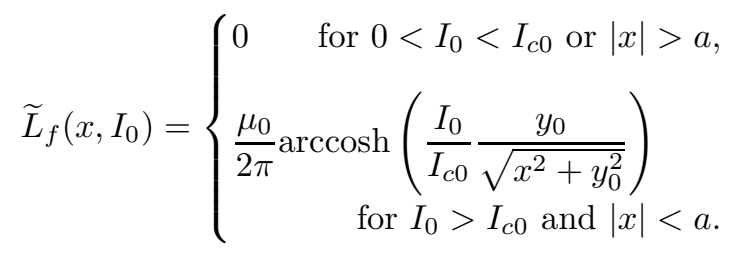

In order to measure $j_{c}$ in superconducting films by the inductive method detecting the harmonic voltages,$\frac{15}{=}$ it is important to estimate the induced electric field in superconducting films ${ }^{23}$ The maximum electric field is induced just below the linear wire at $x=0$, and is given approximately by

$$
\left|E_{f}\right| \leq \frac{\mu_{0}}{\sqrt{2} \pi} \omega I_{c 0}\left(\frac{I_{0}}{I_{c 0}}-1\right)
$$

for $0<I_{0}-I_{c 0} \ll I_{c 0}$.

\section{Harmonic voltages}

The voltage per unit length induced in the linear wire can be expressed as the Fourier series

$$
V(t)=\sum_{n=1}^{\infty} V_{n} \cos \left(n \omega t+\vartheta_{n}\right),
$$

where the amplitude $V_{n}$ and phase difference $\vartheta_{n}$ of the $n$th harmonics are calculated by

$$
V_{n} \exp \left(-i \vartheta_{n}\right)=\frac{1}{\pi} \int_{0}^{2 \pi} d(\omega t) V(t) \exp (i n \omega t) .
$$

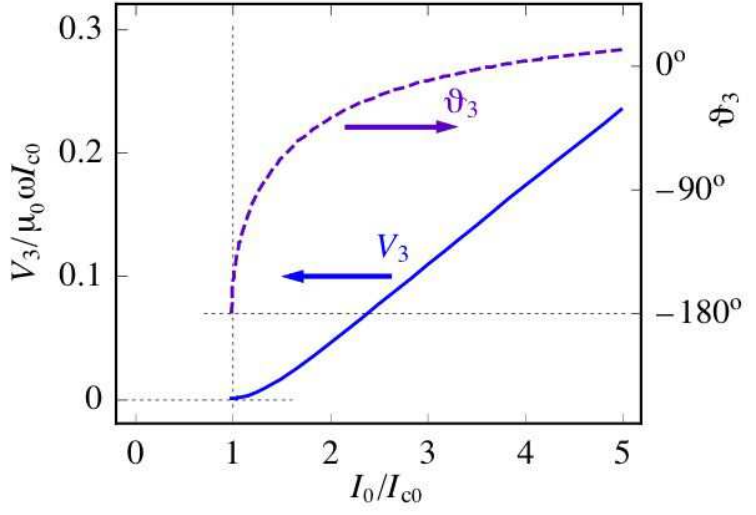

FIG. 3: (color online) The amplitude $V_{3}$ and the phase $\vartheta_{3}$ of the third-harmonic voltage as functions of the drive current amplitude $I_{0}$.

Because of the periodicity of $V(t+\pi / \omega)=-V(t)$, the even harmonics are zero; i.e., $V_{n}=0$ for $n=2,4,6, \ldots$ The fundamental voltage $V_{1} \exp \left(-i \vartheta_{1}\right)$ is simply determined by the resistance per unit length of the linear wire, the self-inductance per unit length of the wire, and the mutual inductance per unit length between the wire and its image. We are interested in the odd harmonics of $V_{n} \exp \left(-i \vartheta_{n}\right)$ for $n=3,5,7, \ldots$; they are obtained by substituting Eqs. (27) and (28) into Eq. (34), which yields

$$
\begin{aligned}
& V_{n} \exp \left(-i \vartheta_{n}\right) \\
& =\frac{2}{\pi} \omega I_{0} \int_{0}^{\pi} d \theta \exp (i n \theta) \sin \theta \widetilde{L}_{w}\left(I_{0}(1-\cos \theta) / 2\right) \\
& =\frac{\mu_{0}}{\pi^{2}} \omega I_{0} \int_{\theta_{c}}^{\pi} d \theta \exp (i n \theta) \sin \theta \ln \left(\frac{I_{0}}{I_{c 0}} \frac{1-\cos \theta}{2}\right),
\end{aligned}
$$

where $\theta_{c}=\arccos \left(1-2 I_{c 0} / I_{0}\right)$. For $0<I_{0} / I_{c 0}-1 \ll 1$ we have

$$
\begin{aligned}
& V_{n} \simeq \frac{\mu_{0}}{\pi^{2}} \omega I_{c 0}\left(\frac{I_{0}}{I_{c 0}}-1\right)^{2}, \\
& \vartheta_{n} \simeq-\pi+\frac{16 n}{15}\left(\frac{I_{0}}{I_{c 0}}-1\right)^{1 / 2} .
\end{aligned}
$$

Figure 3 shows the $I_{0}$ dependence of the thirdharmonic voltage $V_{3} \exp \left(-i \vartheta_{3}\right)$ calculated from Eq. (35) with $n=3$. Although the present model of a linear wire is oversimplified, the behavior of the third harmonic voltage shown in this figure qualitatively agrees with the experimental data measured by a coil with a $\mathrm{YBa}_{2} \mathrm{Cu}_{3} \mathrm{O}_{7-\mathrm{y}}$ film $\underline{15,16,18}$

\section{TWO LINEAR WIRES AND A SUPERCONDUCTING FILM}

Two linear wires are better than a single wire to model current-carrying coils. In this section we consider a su- 
perconducting film and two linear wires carrying transport currents of opposite directions, as shown in Fig. 4 Infinitely long wires, parallel to the $z$ axis, are situated at $(x, y)=\left( \pm x_{0}, y_{0}\right)$ where $x_{0}>0$ and $y_{0}>0 . \mathrm{A}$ superconducting film, infinitely extended in the $x z$ plane, is situated at $-d / 2<y<+d / 2$, where $\lambda<d \ll y_{0}$.

One wire carrying a dc current $+I_{z}$ is situated at $(x, y)=\left(+x_{0}, y_{0}\right)$ and another wire carrying a current of opposite direction $-I_{z}$ is at $(x, y)=\left(-x_{0}, y_{0}\right)$, where $x_{0}>0$ and $y_{0}>0$.

The complex field due to the two wires is given by

$$
\mathcal{H}_{0}(\zeta)=\frac{I_{z}}{2 \pi}\left(\frac{1}{\zeta-\zeta_{1}}-\frac{1}{\zeta-\zeta_{2}}\right)
$$

where $\zeta_{1}=x_{0}+i y_{0}$ and $\zeta_{2}=-x_{0}+i y_{0}=-\zeta_{1}^{*}$.

\section{A. DC response}

In this subsection we consider the magnetic-field distribution when the linear wire carries a dc current $I_{z}=$ $I_{0}>0$ that is held constant after monotonically increasing from $I_{z}=0$.

\section{Linear response for $0<I_{0} \leq I_{c 0}$}

For $0<I_{0} \leq I_{c 0}$, the magnetic field is completely shielded below the film, $y=\operatorname{Im} \zeta<0$. The field distribution can be obtained by the mirror-image technique, and the resulting complex field is

$$
\mathcal{H}(\zeta)= \begin{cases}2 \mathcal{H}_{\|}(\zeta) & \text { for } \operatorname{Im} \zeta>0 \\ 0 & \text { for } \operatorname{Im} \zeta<0\end{cases}
$$

where

$$
\mathcal{H}_{\|}(\zeta)=\frac{I_{0}}{2 \pi}\left(\frac{\zeta}{\zeta^{2}-\zeta_{1}^{2}}-\frac{\zeta}{\zeta^{2}-\zeta_{2}^{2}}\right)
$$

The complex potential for $\operatorname{Im}(\zeta)>0$ is given by

$$
\mathcal{G}(\zeta)=\frac{I_{0}}{2 \pi} \ln \left(\frac{\zeta^{2}-\zeta_{1}^{2}}{\zeta^{2}-\zeta_{2}^{2}}\right)
$$

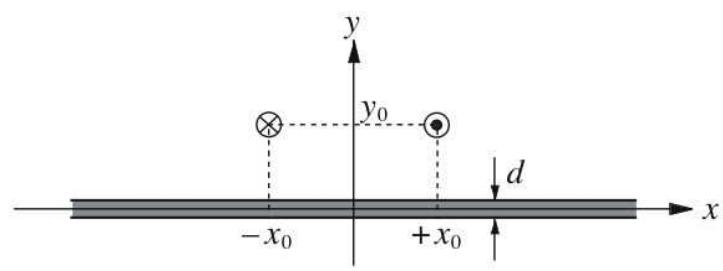

FIG. 4: Configuration of a superconducting film at $y=0$ and two linear wires at $(x, y)=\left( \pm x_{0}, y_{0}\right)$ carrying transport currents $\pm I_{z}$.
The perpendicular magnetic field is $H_{y}(x, 0)=0$, and the sheet-current density is thus given by

$$
K_{z}(x)=-I_{0} F_{w}(x)
$$

where the function $F_{w}(x)$ is determined by the configuration of the wires,

$$
F_{w}(x)=\frac{1}{\pi}\left[\frac{y_{0}}{\left(x-x_{0}\right)^{2}+y_{0}^{2}}-\frac{y_{0}}{\left(x+x_{0}\right)^{2}+y_{0}^{2}}\right] .
$$

The sheet-current density $\left|K_{z}(x)\right|$ is maximum at $x=a_{c}$, where

$$
a_{c}=\sqrt{\frac{1}{3}\left(x_{0}^{2}-y_{0}^{2}+2 \sqrt{x_{0}^{4}+x_{0}^{2} y_{0}^{2}+y_{0}^{4}}\right)} .
$$

The sheet-current density cannot exceed $j_{c} d$; that is, $\left|K_{z}(x)\right| \leq I_{0} F_{w}\left(a_{c}\right) \leq j_{c} d$, and Eq. (42) is valid for $I_{0} \leq I_{c 0}$, where the threshold current is given by

$$
I_{c 0}=j_{c} d / F_{w}\left(a_{c}\right)
$$

Figure 5(a) shows the magnetic-field lines [i.e., the contour lines of $\operatorname{Re} \mathcal{G}(x+i y)$ ] calculated from Eq. (41), and the dashed line in Fig. 5 (d) shows $K_{z}(x)$ given by Eq. (42).

\section{Nonlinear response for $I_{0}>I_{c 0}$}

For $I_{0}>I_{c 0}$, on the other hand, the maximum $\left|K_{z}\right|$ reaches $j_{c} d$ and the magnetic field penetrates below the superconducting film. The field distributions for $I_{0}>$ $I_{c 0}$, therefore, must satisfy

$$
\begin{aligned}
K_{z}(x) & =-j_{c} d \operatorname{sgn}(x) \text { for } b<|x|<a, \\
H_{y}(x, 0) & =0 \text { for }|x|<b \text { or }|x|>a,
\end{aligned}
$$

where the flux fronts are at $x= \pm a$ and $x= \pm b$. The complex field and the parameters $a$ and $b$ (where $a>$ $b>0)$ are determined such that they are consistent with Eqs. (46) and (47), as derived in Appendix C

The parameters for flux fronts, $a$ and $b$, are determined as functions of $I_{0}$ for $I_{0}>I_{c 0}$, by solving the following two equations,

$$
\begin{aligned}
& \frac{I_{0}}{j_{c} d} \operatorname{Re} \sqrt{\frac{\zeta_{1}^{2}-b^{2}}{a^{2}-\zeta_{1}^{2}}}=a \boldsymbol{E}(k)-\frac{b^{2}}{a} \boldsymbol{K}(k), \\
& \frac{I_{0}}{j_{c} d} \operatorname{Re} \sqrt{\frac{a^{2}-\zeta_{1}^{2}}{\zeta_{1}^{2}-b^{2}}}=a[\boldsymbol{K}(k)-\boldsymbol{E}(k)],
\end{aligned}
$$

where $\boldsymbol{K}(k)$ and $\boldsymbol{E}(k)$ are the complete elliptic integrals of the first and second kinds, and $k=\sqrt{1-b^{2} / a^{2}}$. These parameters obey $a=b=a_{c}$ when $I_{0}=I_{c 0}$, and $a>a_{c}>b>0$ when $I_{0}>I_{c 0}$, where $a_{c}$ is given by Eq. (44). As shown in Fig. 6 $a$ increases and $b$ decreases with increasing $I_{0}$. 

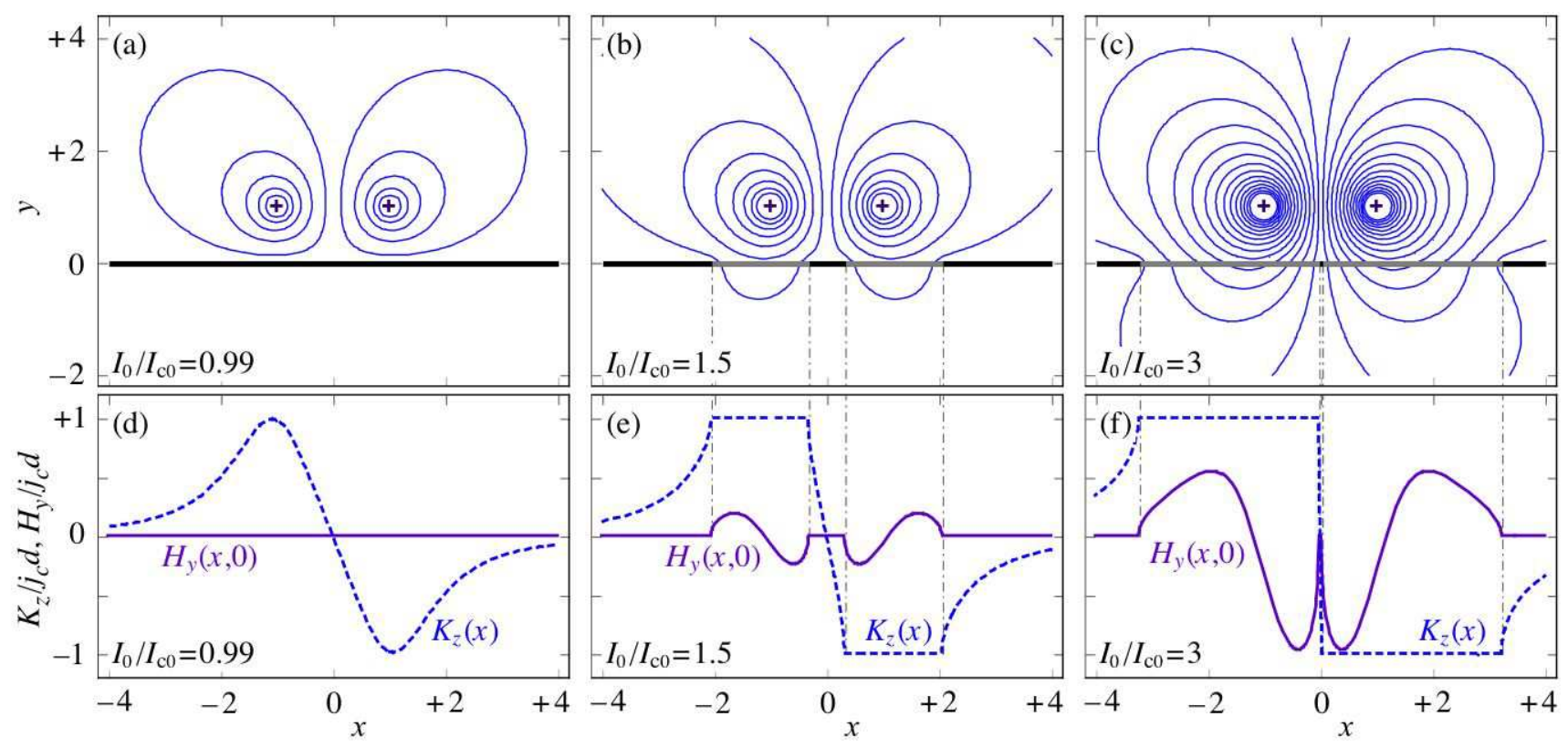

FIG. 5: (color online) Magnetic-field and sheet-current distributions (a,d) for $I_{0} / I_{c 0}=0.99\left(a_{c}=1.07\right)$, (b,e) for $I_{0} / I_{c 0}=1.5$ $(a=2.06$ and $b=0.32)$, and (c,f) for $I_{0} / I_{c 0}=3(a=3.23$ and $b=0.02)$. Top figures (a,b,c) show the magnetic-field lines around linear wires and a superconducting film. The cross symbols at $(x, y)=\left( \pm x_{0}, y_{0}\right)=( \pm 1,1)$ denote the positions of the linear wires, the black horizontal lines the shielded regions $(|x|<b$ or $|x|>a)$ of the superconducting film, and the gray horizontal lines the penetrated regions $b<|x|<a$ of the film. The vertical dot-dashed lines indicate the positions of the flux fronts at $x= \pm a$ and $\pm b$. Bottom figures (d,e,f) show the distributions of the perpendicular magnetic field $H_{y}(x, 0)$ divided by $j_{c} d$ (solid lines) and the sheet-current density $K_{z}(x)$ divided by $j_{c} d$ (dashed lines) in the superconducting film.

The complex field is derived in Appendix [C] and is given by

$$
\mathcal{H}(\zeta)=\mathcal{H}_{\|}(\zeta)+\mathcal{H}_{\perp}(\zeta)+\mathcal{H}_{c}(\zeta)
$$

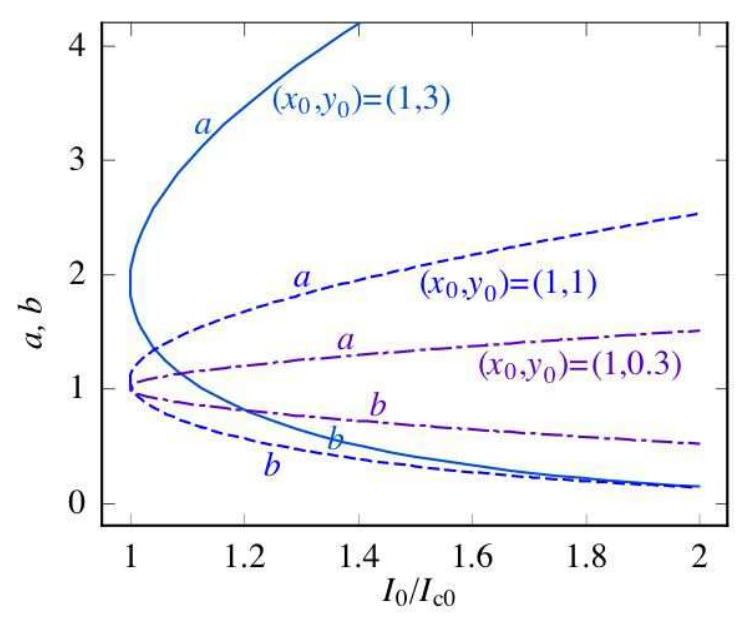

FIG. 6: (color online) The parameters for flux fronts, $a$ (upper lines) and $b$ (lower lines), as functions of $I_{0}$, determined by Eqs. (48) and (49). The solid lines show $a$ and $b$ for $\left(x_{0}, y_{0}\right)=$ $(1,3)\left(a_{c}=1.92\right)$, the dashed lines for $\left(x_{0}, y_{0}\right)=(1,1)\left(a_{c}=\right.$ $1.07)$, and the chained lines for $\left(x_{0}, y_{0}\right)=(1,0.3)\left(a_{c}=1.00\right)$. where

$$
\begin{aligned}
\mathcal{H}_{\perp}(\zeta) & =\frac{I_{0}}{2 \pi} \zeta \phi(\zeta)\left[\frac{1}{\left(\zeta^{2}-\zeta_{1}^{2}\right) \phi\left(\zeta_{1}\right)}-\frac{1}{\left(\zeta^{2}-\zeta_{2}^{2}\right) \phi\left(\zeta_{2}\right)}\right], \\
\mathcal{H}_{c}(\zeta) & =\frac{j_{c} d}{\pi} \zeta \phi(\zeta) \int_{b}^{a} \frac{d u}{\left(u^{2}-\zeta^{2}\right) \phi(u)}, \\
\phi(\zeta) & =\sqrt{\left(a^{2}-\zeta^{2}\right)\left(\zeta^{2}-b^{2}\right)} .
\end{aligned}
$$

Integrating Eq. (50) [or [C9)], we obtain the complex potential $\mathcal{G}(\zeta)=\int \mathcal{H}(\zeta) d \zeta$, as

$$
\begin{aligned}
\mathcal{G}(\zeta)= & \frac{I_{0}}{4 \pi} \ln \left(\frac{\zeta^{2}-\zeta_{1}^{2}}{\zeta^{2}-\zeta_{2}^{2}}\right) \\
& +\frac{I_{0}}{2 \pi}\left[-G_{0}\left(\zeta, \zeta_{1}\right)+G_{0}\left(\zeta, \zeta_{2}\right)\right] \\
& +\frac{j_{c} d}{\pi} \int_{b}^{a} d u G_{0}(\zeta, u),
\end{aligned}
$$

where

$$
G_{0}(\zeta, s)=\operatorname{arctanh} \sqrt{\frac{\zeta^{2}-b^{2}}{a^{2}-\zeta^{2}} \frac{a^{2}-s^{2}}{s^{2}-b^{2}}} .
$$

Figure 5 (b) and (c) show the magnetic-field lines, i.e., the contour lines of $\operatorname{Re} \mathcal{G}(x+i y)$ calculated from Eq. (54). Figure 5(e) and (f) show $H_{y}(x, 0)=\operatorname{Re} \mathcal{H}(x \pm i \epsilon)$ and $K_{z}(x)=\operatorname{Im}[\mathcal{H}(x-i \epsilon)-\mathcal{H}(x+i \epsilon)]$ calculated from Eq. (50) [or from Eqs. (46), (47), (C18), and (C19)]. 


\section{B. AC response}

The response of a superconducting film to ac drive currents $\pm I_{z}(t)= \pm I_{0} \cos \omega t$ flowing in the two linear wires at $(x, y)=\left( \pm x_{0}, y_{0}\right)$ is calculated in a manner similar to that described in Sec. IIB The magnetic flux per unit length $\Phi_{w}$ linked in the two wires is calculated from

$$
\begin{aligned}
\Phi_{w} & =-\mu_{0} \int_{-x_{0}+r_{0}}^{+x_{0}-r_{0}} d x H_{y}\left(x, y_{0}\right) \\
& =\mu_{0} \operatorname{Re}\left[\mathcal{G}\left(\zeta_{2}+r_{0}\right)-\mathcal{G}\left(\zeta_{1}-r_{0}\right)\right],
\end{aligned}
$$

and the magnetic flux per unit length $\Phi_{f}\left(x^{\prime}\right)$ up through the film $(y=0)$ in the region $x^{\prime}<x<+\infty$ is calculated from Eq. (6), where $\mathcal{G}(\zeta)$ is given by Eqs. (41) and (54). The time-dependent magnetic flux is given by Eqs. (26) and (29). The voltage $V(t)$ induced in the wires per unit length and the electric field induced in the film are given by Eqs. (24) and (25), respectively.

\section{DISCUSSION}

Here we discuss the more realistic situation of experiments detecting the response of a superconducting film to a circular current-carrying coil ${ }^{15}$ We consider a singleturn coil parallel to a superconducting film. When the radius of the coil is $x_{0}$ and the distance between the coil and the film is $y_{0}$, the configuration of the coil and the film in the $x y$ plane is similar to that in Fig. 4 We expect that the magnetic-field lines, the magnetic-field component perpendicular to the film, and the circular sheet-current distribution in the film then will be similar to the corresponding quantities shown in Fig. [5

In actual superconducting films the critical current density $j_{c}$ and the film thickness $d$ can be inhomogeneous, although in the present paper we assumed that $j_{c} d$ is spatially homogeneous. The magnetic field produced by the coil of radius $x_{0}$ is largest directly below the coil (i.e., the circular region of radius $\sim x_{0}$ in the film) when $x_{0} \gtrsim y_{0}$. If $j_{c} d$ in the circular region below the coil is nearly homogeneous, the response of the film will be similar to that presented in Sec.III and the signal in the coil will yield the local value of $j_{c} d$. In this sense the resolution of the measurements using the coil is on the order of $x_{0}$. However, if $j_{c} d$ is very inhomogeneous within the circular region below the coil, the response of the film will be quite different from that given in Sec. III]

\section{CONCLUSION}

We investigated the response of an infinite superconducting film at $y=0$ to linear wires above the film carrying transport currents $I_{z}$. We derived analytic expressions for the complex field, and these are given in
Eqs. (17), (16), (39), and (50). The behavior of the film can be summarized as follows.

(i) Response to a dc current, $I_{z}=I_{0}$ : For a small dc current in the range $0<I_{0}<I_{c 0} \propto j_{c} d$, the sheet-current density $K_{z}$ in the film is less than the critical value $j_{c} d$, and no magnetic field penetrates into the region $y<0$ below the film. However, for a large dc current $I_{0}>$ $I_{c 0}$, the sheet-current density reaches $\left|K_{z}\right|=j_{c} d$ and the magnetic field penetrates into the region below the film.

(ii) Response to an ac current, $I_{z}=I_{0} \cos \omega t$ : For $0<I_{0}<I_{c 0}$ the voltage $V(t)$ induced in the linear wire has only the first harmonic at the fundamental frequency $\omega_{1}=\omega$. However, for $I_{0}>I_{c 0}$, odd-harmonic voltages with frequency $\omega_{n}=n \omega, n=3,5,7, \ldots$, are also induced in the linear wire.

The complex field for the case of a single currentcarrying linear wire above the film is derived in Appendix A. The complex field for the case of a pair of currentcarrying wires in a plane perpendicular (parallel) to the film is given in Appendix B (Appendix C).

\section{ACKNOWLEDGMENTS}

We thank H. Yamasaki for stimulating discussions. This manuscript has been authored in part by Iowa State University of Science and Technology under Contract No. W-7405-ENG-82 with the U.S. Department of Energy.

\section{APPENDIX A: COMPLEX FIELD FOR A SUPERCONDUCTING FILM WITH A LINEAR CURRENT-CARRYING WIRE ABOVE IT}

In this appendix we derive Eqs. (15) and (16). Consider the function $f(\zeta)$ defined by

$$
f(\zeta)=\left[\mathcal{H}(\zeta)-\eta_{\|}(\zeta)\right] \sqrt{a^{2}-\zeta^{2}},
$$

where $\eta_{\|}(\zeta)$ is

$$
\eta_{\|}(\zeta)=\frac{I_{0}}{2 \pi} \frac{i y_{0}}{\zeta^{2}+y_{0}^{2}} .
$$

We calculate the integral $\oint_{C} d \zeta^{\prime} f\left(\zeta^{\prime}\right) /\left(\zeta^{\prime}-\zeta\right)$ along the closed contour $C$ shown in Fig. 7 as

$$
\frac{1}{2 \pi i} \oint_{C} d \zeta^{\prime} \frac{f\left(\zeta^{\prime}\right)}{\zeta^{\prime}-\zeta}=\frac{1}{2 \pi i} \int_{-\infty}^{+\infty} d u \frac{f(u+i \epsilon)-f(u-i \epsilon)}{u-\zeta}
$$

where the contour integral along the infinite circle vanishes because $|f(\zeta)| \sim|\mathcal{H}(\zeta) \zeta| \rightarrow 0$ for $|\zeta| \rightarrow \infty$. Because the integrand in the left-hand side of Eq. (A3) has poles at $\zeta^{\prime}=\zeta$ and $\zeta^{\prime}= \pm i y_{0}$, the contour integral of Eq. A3 is also calculated by using the residue theorem, as $f(\zeta)-f_{0}(\zeta)$, and thus yielding

$$
f(\zeta)-f_{0}(\zeta)=\frac{1}{2 \pi i} \int_{-\infty}^{+\infty} d u \frac{f(u+i \epsilon)-f(u-i \epsilon)}{u-\zeta},
$$




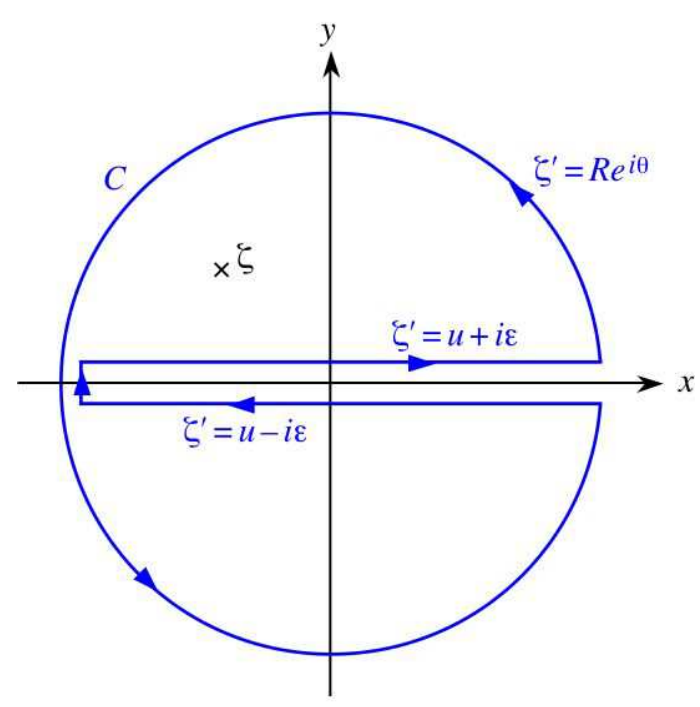

FIG. 7: (color online) The contour $C$ in the $\zeta^{\prime}$ plane for the integrals in Eqs. A3 and (C2) consists of a line just above the real axis, $\zeta^{\prime}=u+i \epsilon$ from $u=-R^{\prime}$ to $u=+R$ where $R^{\prime}<R$, an infinite circle, $\zeta^{\prime}=R e^{i \theta}$ from $\theta=\epsilon / R$ to $\theta=2 \pi-\epsilon / R$, a line just below the real axis, $\zeta^{\prime}=u-i \epsilon$ from $u=+R$ to $u=-R^{\prime}$, and an infinitesimal line parallel to the imaginary axis, $\zeta^{\prime}=-R^{\prime}+i v$ from $v=-\epsilon$ to $v=+\epsilon$, taking the limit of $R \rightarrow \infty, R^{\prime} \rightarrow \infty$, and $\epsilon \rightarrow+0$.

where $f_{0}(\zeta)$ is given by

$$
f_{0}(\zeta)=\frac{I_{0}}{2 \pi} \frac{\zeta \sqrt{a^{2}+y_{0}^{2}}}{\zeta^{2}+y_{0}^{2}} .
$$

For $\zeta=x \pm i \epsilon$, the $\mathcal{H}(\zeta)$ and $\eta_{\|}(\zeta)$ are given by

$$
\begin{aligned}
\mathcal{H}(x \pm i \epsilon) & =H_{y}(x, 0)+i\left[\operatorname{Im} \mathcal{H}_{0}(x) \mp K_{z}(x) / 2\right], \\
\eta_{\|}(x) & =i \operatorname{Im} \mathcal{H}_{0}(x)
\end{aligned}
$$

respectively, and thus yielding

$$
\mathcal{H}(x \pm i \epsilon)-\eta_{\|}(x)=H_{y}(x, 0) \mp i K_{z}(x) / 2 .
$$

By using Eqs. (A1), and

$$
\sqrt{a^{2}-(x \pm i \epsilon)^{2}}= \begin{cases}\mp i \operatorname{sgn}(x) \sqrt{x^{2}-a^{2}} & \text { for }|x|>a, \\ \sqrt{a^{2}-x^{2}} & \text { for }|x|<a,\end{cases}
$$

we have

$$
\begin{aligned}
& f(x+i \epsilon)-f(x-i \epsilon) \\
& = \begin{cases}-2 i \operatorname{sgn}(x) \sqrt{x^{2}-a^{2}} H_{y}(x, 0) & \text { for }|x|>a, \\
-i \sqrt{a^{2}-x^{2}} K_{z}(x) & \text { for }|x|<a,\end{cases} \\
& = \begin{cases}0 & \text { for }|x|>a, \\
i \sqrt{a^{2}-x^{2}} j_{c} d & \text { for }|x|<a,\end{cases}
\end{aligned}
$$

where we used Eqs. (13) and (14). Substitution of Eq. (A10) into Eq. (A4) yields

$$
\begin{aligned}
f(\zeta)-f_{0}(\zeta) & =\frac{j_{c} d}{2 \pi} \int_{-a}^{+a} d u \frac{\sqrt{a^{2}-u^{2}}}{u-\zeta} \\
& =\frac{j_{c} d}{2}\left(-\zeta \pm i \sqrt{a^{2}-\zeta^{2}}\right)
\end{aligned}
$$

where the upper (lower) signs hold for $\operatorname{Im} \zeta>0(\operatorname{Im} \zeta<$ $0)$.

From Eqs. A1, A5, and A11 we obtain

$$
\begin{aligned}
\mathcal{H}(\zeta)= & \frac{I_{0}}{2 \pi} \frac{1}{\zeta^{2}+y_{0}^{2}}\left(i y_{0}+\frac{\zeta \sqrt{a^{2}+y_{0}^{2}}}{\sqrt{a^{2}-\zeta^{2}}}\right) \\
& +\frac{j_{c} d}{2}\left( \pm i-\frac{\zeta}{\sqrt{a^{2}-\zeta^{2}}}\right) .
\end{aligned}
$$

Note that the first term of the right-hand side of Eq. A12, which is proportional to $I_{0}$, corresponds to the complex field in the ideal Meissner state for two semi-infinite strips situated at $|\operatorname{Re} \zeta|>a$ with a line current at $\zeta=i y_{0}$. By using $\sqrt{-\zeta^{2}}=\mp i \zeta$, we confirm that Eq. (A12) for $a \rightarrow 0$ is identical to Eq. (7). Equation (A12) can be rewritten as

$$
\begin{aligned}
\mathcal{H}(\zeta)= & \pm i \frac{j_{c} d}{2}+\frac{I_{0}}{2 \pi} \frac{1}{\zeta^{2}+y_{0}^{2}}\left(i y_{0}+\frac{\zeta \sqrt{a^{2}-\zeta^{2}}}{\sqrt{a^{2}+y_{0}^{2}}}\right) \\
& +\frac{\zeta}{\sqrt{a^{2}-\zeta^{2}}}\left(\frac{I_{0}}{2 \pi} \frac{1}{\sqrt{a^{2}+y_{0}^{2}}}-\frac{j_{c} d}{2}\right) \cdot(\mathrm{A} 13)
\end{aligned}
$$

However, the last term of the right-hand side of Eq. A13) must vanish, because $\mathcal{H}(\zeta)$ is finite at $\zeta= \pm a$. We thus obtain $I_{0}=\pi j_{c} d \sqrt{a^{2}+y_{0}^{2}}$, such that the parameter $a$ describing the position of the flux front is given by Eq. (15). The resulting expression for the complex field is given by Eq. (16).

See also Appendix B for the complex field for a superconducting film with two wires in the $y z$ plane.

\section{APPENDIX B: COMPLEX FIELD FOR TWO PARALLEL WIRES IN THE $y z$ PLANE}

We present here the complex field for a superconducting film with two parallel linear wires above it. An infinite superconducting film is in the plane $y=0$, as shown in Fig. 10 One wire carrying a dc current $+I_{0}$ is situated at $(x, y)=\left(0, y_{1}\right)$ and another wire carrying current $-I_{0}$ is at $(x, y)=\left(0, y_{2}\right)$, where $0<y_{1}<y_{2}$. The derivation of the complex field for the two wires is similar to that given in Appendix $\mathrm{A}$ for a single wire, and we exhibit here only the resulting expressions.

For $0<I_{0}<I_{c 0}$, the sheet-current density and the perpendicular magnetic field satisfy $\left|K_{z}(x)\right|<j_{c} d$ and 
$H_{y}(x, 0)=0$, respectively. The threshold current is given by

$$
I_{c 0}=\pi j_{c} d \frac{y_{1} y_{2}}{y_{2}-y_{1}}
$$

and the complex field for $0<I_{0}<I_{c 0}$ is

$$
\mathcal{H}(\zeta)= \begin{cases}\frac{I_{0}}{\pi}\left(\frac{i y_{1}}{\zeta^{2}+y_{1}^{2}}-\frac{i y_{2}}{\zeta^{2}+y_{2}^{2}}\right) & \text { for } \operatorname{Im} \zeta>0 \\ 0 & \text { for } \operatorname{Im} \zeta<0\end{cases}
$$

For $I_{0}>I_{c 0}, K_{z}(x)$ and $H_{y}(x, 0)$ fulfill Eqs. (13) and (14), and the relationship between $a$ and $I_{0}$ is given by

$$
I_{0}=\pi j_{c} d\left(\frac{1}{\sqrt{a^{2}+y_{1}^{2}}}-\frac{1}{\sqrt{a^{2}+y_{2}^{2}}}\right)^{-1} .
$$

The complex field and complex potential for $I_{0}>I_{c 0}$ are respectively given by

$$
\begin{aligned}
\mathcal{H}(\zeta)= & \pm i \frac{j_{c} d}{2}+\frac{I_{0}}{2 \pi}\left[\frac{1}{\zeta^{2}+y_{1}^{2}}\left(i y_{1}+\frac{\zeta \sqrt{a^{2}-\zeta^{2}}}{\sqrt{a^{2}+y_{1}^{2}}}\right)\right. \\
& \left.-\frac{1}{\zeta^{2}+y_{2}^{2}}\left(i y_{2}+\frac{\zeta \sqrt{a^{2}-\zeta^{2}}}{\sqrt{a^{2}+y_{2}^{2}}}\right)\right], \\
\mathcal{G}(\zeta)= & \frac{j_{c} d}{2}\left( \pm i \zeta+\sqrt{a^{2}-\zeta^{2}}\right) \\
& +\frac{I_{0}}{2 \pi}\left[i \arctan \left(\frac{\zeta}{y_{1}}\right)-\operatorname{arctanh}\left(\sqrt{\frac{a^{2}-\zeta^{2}}{a^{2}+y_{1}^{2}}}\right)\right. \\
& \left.-i \arctan \left(\frac{\zeta}{y_{2}}\right)+\operatorname{arctanh}\left(\sqrt{\frac{a^{2}-\zeta^{2}}{a^{2}+y_{2}^{2}}}\right)\right] .
\end{aligned}
$$

\section{APPENDIX C: COMPLEX FIELD FOR TWO PARALLEL WIRES IN A PLANE PARALLEL TO THE $x z$ PLANE}

In this appendix we derive Eqs. (48)-(52). Consider the function $F(\zeta)$ defined by

$$
F(\zeta)=\left[\mathcal{H}(\zeta)-\mathcal{H}_{\|}(\zeta)\right] \phi(\zeta)
$$

where $\mathcal{H}_{\|}(\zeta)$ and $\phi(\zeta)$ are defined by Eqs. (40) and (53), respectively. We calculate the integral $\oint_{C} d \zeta^{\prime} F\left(\zeta^{\prime}\right) /\left(\zeta^{\prime}-\right.$ $\zeta$ ) along the closed contour $C$ shown in Fig. [7] as in Eq. A3),

$$
\frac{1}{2 \pi i} \oint_{C} d \zeta^{\prime} \frac{F\left(\zeta^{\prime}\right)}{\zeta^{\prime}-\zeta}=\frac{1}{2 \pi i} \int_{-\infty}^{+\infty} d u \frac{F(u+i \epsilon)-F(u-i \epsilon)}{u-\zeta}
$$

where the contour integral along the infinite circle vanishes because $|F(\zeta)| \sim\left|\mathcal{H}(\zeta) \zeta^{2}\right| \rightarrow 0$ for $|\zeta| \rightarrow \infty$. Because the integrand on the left-hand side of Eq. (C2) has poles at $\zeta^{\prime}=\zeta, \pm \zeta_{1}$, and $\pm \zeta_{2}$, the contour integral of
Eq. (C2) can be calculated by using the residue theorem, as $F(\zeta)-F_{0}(\zeta)$, and thus yielding

$$
F(\zeta)-F_{0}(\zeta)=\frac{1}{2 \pi i} \int_{-\infty}^{+\infty} d u \frac{F(u+i \epsilon)-F(u-i \epsilon)}{u-\zeta}
$$

The $F_{0}(\zeta)$ is defined by

$$
\begin{aligned}
F_{0}(\zeta) & =\frac{I_{0}}{4 \pi}\left[\frac{\phi\left(\zeta_{1}\right)}{\zeta-\zeta_{1}}-\frac{\phi\left(\zeta_{2}\right)}{\zeta-\zeta_{2}}-\frac{\phi\left(-\zeta_{1}\right)}{\zeta+\zeta_{1}}+\frac{\phi\left(-\zeta_{2}\right)}{\zeta+\zeta_{2}}\right] \\
& =\frac{I_{0}}{2 \pi}\left[\frac{\zeta \phi\left(\zeta_{1}\right)}{\zeta^{2}-\zeta_{1}^{2}}-\frac{\zeta \phi\left(\zeta_{2}\right)}{\zeta^{2}-\zeta_{2}^{2}}\right],
\end{aligned}
$$

where we used $\phi\left(-\zeta_{1}\right)=-\phi\left(\zeta_{1}\right)$ and $\phi\left(-\zeta_{2}\right)=-\phi\left(\zeta_{2}\right)$.

For $\zeta=x \pm i \epsilon$, Eq. (53) is reduced to

$$
\phi(x \pm i \epsilon)= \begin{cases} \pm i \widetilde{\phi}(x) & \text { for }|x|<b \text { or }|x|>a \\ \operatorname{sgn}(x) \widetilde{\phi}(x) & \text { for } b<|x|<a\end{cases}
$$

where

$$
\begin{aligned}
\widetilde{\phi}(x)= & \operatorname{sgn}(a-|x|) \sqrt{\left|\left(a^{2}-x^{2}\right)\left(x^{2}-b^{2}\right)\right|} \\
= & \begin{cases}\sqrt{\left(a^{2}-x^{2}\right)\left(b^{2}-x^{2}\right)} & \text { for }|x|<b, \\
\sqrt{\left(a^{2}-x^{2}\right)\left(x^{2}-b^{2}\right)} & \text { for } b<|x|<a, \\
-\sqrt{\left(x^{2}-a^{2}\right)\left(x^{2}-b^{2}\right)} & \text { for }|x|>a .\end{cases}
\end{aligned}
$$

Substituting Eqs. (A8) and (C5) into Eq. (C1) with $\zeta=$ $x \pm i \epsilon$, we have

$$
\begin{aligned}
& F(x+i \epsilon)-F(x-i \epsilon) \\
& = \begin{cases}2 i \widetilde{\phi}(x) H_{y}(x, 0) & \text { for }|x|<b \text { or }|x|>a, \\
-i \operatorname{sgn}(x) \widetilde{\phi}(x) K_{z}(x) & \text { for } b<|x|<a,\end{cases} \\
& = \begin{cases}0 & \text { for }|x|<b \text { or }|x|>a, \\
i j_{c} d \widetilde{\phi}(x) & \text { for } b<|x|<a,\end{cases}
\end{aligned}
$$

where we used Eqs. (46) and (47). Substitution of Eq. (C7) into Eq. (C3) yields

$$
\begin{aligned}
F(\zeta)-F_{0}(\zeta) & =\frac{j_{c} d}{2 \pi} \int_{b<|u|<a} d u \frac{\widetilde{\phi}(u)}{u-\zeta} \\
& =\frac{j_{c} d}{\pi} \int_{b}^{a} d u \frac{\zeta \phi(u)}{u^{2}-\zeta^{2}}
\end{aligned}
$$

such that

$$
\mathcal{H}(\zeta)=\mathcal{H}_{\|}(\zeta)+\frac{F_{0}(\zeta)}{\phi(\zeta)}+\frac{j_{c} d}{\pi} \frac{\zeta}{\phi(\zeta)} \int_{b}^{a} d u \frac{\phi(u)}{u^{2}-\zeta^{2}}
$$

By using

$$
\frac{\phi(s)}{\phi(\zeta)}=\frac{\phi(\zeta)}{\phi(s)}+\frac{\left(\zeta^{2}-s^{2}\right)\left(\zeta^{2}+s^{2}-a^{2}-b^{2}\right)}{\phi(\zeta) \phi(s)}
$$


we rewrite Eq. as as

$$
\begin{gathered}
\mathcal{H}(\zeta)-\mathcal{H}_{\|}(\zeta) \\
=\zeta \phi(\zeta)\left\{\frac{I_{0}}{2 \pi}\left[\frac{1}{\left(\zeta^{2}-\zeta_{1}^{2}\right) \phi\left(\zeta_{1}\right)}-\frac{1}{\left(\zeta^{2}-\zeta_{2}^{2}\right) \phi\left(\zeta_{2}\right)}\right]\right. \\
\left.+\frac{j_{c} d}{\pi} \int_{b}^{a} \frac{d u}{\left(u^{2}-\zeta^{2}\right) \phi(u)}\right\} \\
+\frac{\zeta}{\phi(\zeta)} \mathcal{R}(\zeta)
\end{gathered}
$$

where

$$
\begin{aligned}
\mathcal{R}(\zeta)= & \frac{I_{0}}{2 \pi}\left[\frac{\zeta^{2}+\zeta_{1}^{2}-a^{2}-b^{2}}{\phi\left(\zeta_{1}\right)}-\frac{\zeta^{2}+\zeta_{2}^{2}-a^{2}-b^{2}}{\phi\left(\zeta_{2}\right)}\right] \\
& -\frac{j_{c} d}{\pi} \int_{b}^{a} d u \frac{\zeta^{2}+u^{2}-a^{2}-b^{2}}{\widetilde{\phi}(u)} \\
= & \frac{\left(\zeta^{2}-b^{2}\right) \mathcal{R}( \pm a)+\left(a^{2}-\zeta^{2}\right) \mathcal{R}( \pm b)}{a^{2}-b^{2}}
\end{aligned}
$$

For $\zeta= \pm a$ and $\pm b$, we have

$$
\begin{aligned}
\mathcal{R}( \pm a)= & \frac{I_{0}}{2 \pi}\left(\sqrt{\frac{\zeta_{1}^{2}-b^{2}}{a^{2}-\zeta_{1}^{2}}}-\sqrt{\frac{\zeta_{2}^{2}-b^{2}}{a^{2}-\zeta_{2}^{2}}}\right) \\
& -\frac{j_{c} d}{\pi} \int_{b}^{a} d u \sqrt{\frac{u^{2}-b^{2}}{a^{2}-u^{2}}}, \\
\mathcal{R}( \pm b)= & \frac{I_{0}}{2 \pi}\left(\sqrt{\frac{a^{2}-\zeta_{1}^{2}}{\zeta_{1}^{2}-b^{2}}}-\sqrt{\frac{a^{2}-\zeta_{2}^{2}}{\zeta_{2}^{2}-b^{2}}}\right) \\
& -\frac{j_{c} d}{\pi} \int_{b}^{a} d u \sqrt{\frac{a^{2}-u^{2}}{u^{2}-b^{2}}} .
\end{aligned}
$$

In order to remove the divergences in Eq. C11 at $\zeta= \pm a$ and $\pm b$, we require $\mathcal{R}( \pm a)=\mathcal{R}( \pm b)=0$, thus yielding

$$
\begin{aligned}
& \frac{I_{0}}{j_{c} d} \operatorname{Re} \sqrt{\frac{\zeta_{1}^{2}-b^{2}}{a^{2}-\zeta_{1}^{2}}}=\int_{b}^{a} d u \sqrt{\frac{u^{2}-b^{2}}{a^{2}-u^{2}}} \\
& \frac{I_{0}}{j_{c} d} \operatorname{Re} \sqrt{\frac{a^{2}-\zeta_{1}^{2}}{\zeta_{1}^{2}-b^{2}}}=\int_{b}^{a} d u \sqrt{\frac{a^{2}-u^{2}}{u^{2}-b^{2}}}
\end{aligned}
$$

Equations (C15) and (C16) reduce to Eqs. (48) and (49), respectively. We thus obtain $\mathcal{R}(\zeta)=0$ for any $\zeta$, and Eq. (C11) is reduced to Eqs. (50), (51), and (52).

The sheet current $K_{z}(x)=\operatorname{Im}[\mathcal{H}(x-i \epsilon)-\mathcal{H}(x+$ $i \epsilon)$ ] and the perpendicular magnetic field $H_{y}(x, 0)=$ $\operatorname{Re} \mathcal{H}(x \pm i \epsilon)$ are obtained by substituting $\zeta=x \pm i \epsilon$ in Eq. (50) with Eq. (A8),

$$
\begin{gathered}
H_{y}(x, 0) \mp i K_{z}(x) / 2=\mathcal{H}(x \pm i \epsilon)-\mathcal{H}_{\|}(x) \\
=\phi(x \pm i \epsilon)\left\{\frac{I_{0}}{2 \pi}\left[\frac{x}{\left(x^{2}-\zeta_{1}^{2}\right) \phi\left(\zeta_{1}\right)}-\frac{x}{\left(x^{2}-\zeta_{2}^{2}\right) \phi\left(\zeta_{2}\right)}\right]\right. \\
\left.-\frac{j_{c} d}{\pi} \int_{b}^{a} \frac{d u}{\left[(x \pm i \epsilon)^{2}-u^{2}\right] \phi(u)}\right\} \\
=\phi(x \pm i \epsilon)\left\{\frac{I_{0}}{\pi} \operatorname{Re}\left[\frac{x}{\left(x^{2}-\zeta_{1}^{2}\right) \phi\left(\zeta_{1}\right)}\right]\right. \\
-\frac{j_{c} d}{\pi} \int_{b}^{a} \frac{d u}{\phi(u)}\left[\frac{x}{x^{2}-u^{2}}\right. \\
\left.\left.\mp \frac{i \pi}{2}(\delta(x-u)+\delta(x+u))\right]\right\} .
\end{gathered}
$$

Substitution of Eq. C5 into Eq. C17 yields the sheet current $K_{z}(x)$ and the perpendicular magnetic field $H_{y}(x, 0)$. The $K_{z}(x)$ for $b<|x|<a$ is given by Eq. (46), and that for $|x|<b$ or $|x|>a$ is given by

$$
\begin{aligned}
K_{z}(x)= & \frac{2}{\pi} x \widetilde{\phi}(x)\left\{I_{0} \operatorname{Re}\left[\frac{1}{\left(x^{2}-\zeta_{1}^{2}\right) \phi\left(\zeta_{1}\right)}\right]\right. \\
& \left.-j_{c} d \int_{b}^{a} \frac{d u}{\left(x^{2}-u^{2}\right) \phi(u)}\right\} .
\end{aligned}
$$

The $H_{y}(x, 0)$ for $|x|<b$ or $|x|>a$ is given by Eq. (47), and that for $b<|x|<a$ is given by

$$
\begin{aligned}
H_{y}(x, 0)= & \frac{1}{\pi}|x| \widetilde{\phi}(x)\left\{I_{0} \operatorname{Re}\left[\frac{1}{\left(x^{2}-\zeta_{1}^{2}\right) \phi\left(\zeta_{1}\right)}\right]\right. \\
& \left.-j_{c} d \mathrm{P} \int_{b}^{a} \frac{d u}{\left(x^{2}-u^{2}\right) \phi(u)}\right\},
\end{aligned}
$$

where $\mathrm{P}$ denotes the Cauchy principal value integral.
1 G. W. Swan, J. Math. Phys. 9, 1308 (1968).

2 M. R. Halse, J. Phys. D 3, 717 (1970).

${ }^{3}$ P. N. Mikheenko and Yu. E. Kuzovlev, Physica C 204, 229 (1993).

4 J. Zhu, J. Mester, J. Lockhart and J. Turneaure, Physica C 212, 216 (1993).

5 E. H. Brandt, M. V. Indenbom and A. Forkl, Europhys Lett. 22, 735 (1993); E. H. Brandt and M. Indenbom, Phys. Rev. B 48, 12893 (1993).

6 E. Zeldov, J. R. Clem, M. McElfresh and M. Darwin, Phys. Rev. B 49, 9802 (1994).

7 C. P. Bean, Phys. Rev. Lett. 8, 250 (1962); Rev. Mod. Phys. 36, 31 (1964).
8 A. T. Fiory, A. F. Hebard, P. M. Mankiewich, and R. E. Howard, Appl. Phys. Lett. 52, 2165 (1988).

9 J. R. Clem and M. W. Coffey, Phys. Rev. B 46, 14662 (1992).

10 Th. Klupsch and M. Zeisberger, Physica C 244, 153 (1995).

11 J. Gilchrist and E. H. Brandt, Phys. Rev. B 54, 3530 (1996).

12 S. J. Turneaure, A. A. Pesetski, and T. R. Lemberger, J. Appl. Phys. 83, 4334, (1998).

13 M. W. Coffey, J. Appl. Phys. 89, 5570 (2001).

${ }^{14}$ K. Bernhardt, R. Gross, M. Hartmann, R. P. Huebener, F. Kober, D. Koelle, and T. Sermet, Physica C 161, 468 
(1989).

15 J. H. Claassen, M. E. Reeves, and R. J. Soulen, Jr., Rev. Sci. Instrum. 62, 996 (1991).

16 G. D. Poulin, J. S. Preston, and T. Strach, Phys. Rev. B 48, 1077 (1993).

17 H. Hochmuth and M. Lorenz, Physica C 220, 209 (1994); ibid. 265, 335 (1996).

18 Y. Mawatari, H. Yamasaki, and Y. Nakagawa, Appl. Phys. Lett. 81, 2424 (2002).

19 L. S. Koo and K. L. Telschow, Phys. Rev. B 53, 8743 (1996).

${ }^{20}$ H. Wada, M. Migita, E. S. Otabe, M. Kiuchi, T. Matsushita, Y. Mawatari, H. Yamasaki, and Y. Nakagawa, Physica C 392-396, 1310 (2003); T. Nadami, E. S. Otabe, M. Kiuchi, and T. Matsushita, ibid. 412-414, 1011 (2004); T. Nadami, E. S. Otabe, M. Kiuchi, T. Matsushita, Y. Mawatari, H. Yamasaki, and Y. Nakagawa, ibid. 426-431, 688 (2005).

${ }^{21}$ H. Yamada, A. Bitoh, Y. Mitsuno, I. Imai, K. Nomura, K. Kanayama, S. Nakagawa, Y. Mawatari, and H. Yamasaki, Physica C 433, 59 (2005).

${ }^{22}$ M. Aurino, E. Di Gennaro, F. Di Iorio, A. Gauzzi, G. Lamura, and A. Andreone, J. Appl. Phys. 98, 123901 (2005).

23 H. Yamasaki, Y. Mawatari, and Y. Nakagawa, Appl. Phys. Lett. 82, 3275 (2003).

24 The flux-penetration process for finite $H_{c 1} / j_{c} d>0$ is more complicated than that shown in the present paper for $H_{c 1} / j_{c} d \rightarrow 0$. The case for $H_{c 1} / j_{c} d>0$ will be published elsewhere, J. R. Clem and Y. Mawatari, unpublished.

25 J. R. Clem, R. P. Huebener, and D. E. Gallus, J. Low Temp. Phys. 12, 449 (1973).

26 E. Zeldov, A. I. Larkin, V. B. Geshkenbein, M. Konczykowski, D. Majer, B. Khaykovich, V. M. Vinokur, and H. Shtrikman, Phys. Rev. Lett. 73, 1428 (1994).

27 Y. Mawatari and J. R. Clem, Phys. Rev. Lett. 86, 2870 (2001); A. A. Babaei Brojeny, Y. Mawatari, M. Benkraouda, and J. R. Clem, Supercond. Sci. Technol. 15, 1454 (2002); Y. Mawatari and J. R. Clem, Phys. Rev. B 68, 024505 (2003).

28 Note that the simple result of $\Phi_{f}=E_{f}=0$ for $0<I_{0}<$ $I_{c 0}$ is ideally accurate only for the thin-film limit, $d \rightarrow 0$. Small but finite magnitudes of $\left|\Phi_{f}\right|$ and $\left|E_{f}\right|$ arise even for $0<I_{0}<I_{c 0}$ in a superconducting film of finite thickness, because the magnetic flux penetrates in the vicinity of the upper surface of the film.

${ }^{29}$ We confine our attention here to low frequencies, well below those normally used to investigate the surface resistance of superconducting films, where energy dissipation arises due to excitation of quasiparticles or to smallamplitude vortex motion, as discussed, for example, in M. W. Coffey and J. R. Clem, Phys. Rev. Lett. 67, 386 (1991). 